\title{
Les processus de sandhi externe en français de Marseille
}

\author{
JEAN-PHILIPPE WATBLED \\ Université de Provence \\ (Received I9 April 1990)
}

\section{ABSTRACT}

L'objet de cet article est l'étude des processus d'élision et de liaison en français de Marseille. Une attention partiçulière est accordée aux mots avec initiale vocalique ou semi-vocalique qui bloquent ces processus. La recherche des principes qui régissent leur application dans le parler en question révèle un certain nombre de régularités. On est ainsi amené à considérer le $\mathrm{H}$ aspiré comme une position démarcative plutôt qu'une position phonémique vide. D'autre part, on constate une corrélation nette et non fortuite entre le comportement des séquences semi-voyelle + voyelle (à l'initiale du mot) en contexte de liaison/élision, les règles de construction de la syllabe, et les contraintes distributionnelles. La découverte de cette corrélation permet de déterminer quelles règles productives sont à l'oeuvre en discours aux frontières de mot. Elle conduit aussi à une révision de l'analyse de la structure syllabique.

\section{O. INTRODUCTION}

L'objet de cet article est l'examen de deux processus de sandhi externe en français de Marseille: la liaison et l'élision. Ces processus sont bien connus (voir par exemple Schane (I968), Love (I98I), Tranel (I98I), Durand (I986), Encrevé (1988)), et concernent en fait toutes les variétés de français.

Rappelons - en termes traditionnels - qu'il y a liaison en français quand la consonne graphique finale d'un mot est prononcée avant voyelle, alors qu' elle est muette avant consonne ou pause: ainsi le ' $T$ ' final de petit est muet dans le petit garcon: [løpøtigass̃̃], mais prononcé dans le petit ami [løpøtitami]; il y a élision quand le 'E' final de mot tombe avant une voyelle, comme dans l'enfant (=le+enfant): [lãfã] (voir Léon, I966: I I). En outre liaison et élision ont lieu avant certains mots à initiale semi-vocalique (oiseau, par exemple), mais pas avant d'autres (ouistiti, par exemple), et enfin, les mots dits à $\mathrm{H}$ aspiré (héros, honte, etc.: voir plus loin), bien qu'ayant une initiale vocalique d'un point de vue strictement.phonétique, empêchent les deux processus de s'appliquer.

Nous nous proposons ici de déterminer dans quels contextes phonétiques 
la liaison et l'élision ont lieu en français de Marseille, et de voir s'il est possible de formuler des principes généraux permettant de prédire leurs conditions d'application. Nous porterons plus spécialement notre attention sur les mots à initiale vocalique ou semi-vocalique qui empêchent la liaison et l'élision de s'appliquer.

Les données sont essentiellement le résultat d'observations régulières effectuées ces dernières années lors de conversations informelles au cours desquelles les locuteurs ${ }^{1}$ se sont exprimés sans surveiller leur discours. Précisions que tous ces locuteurs sont nés à Marseille ou à proximité de Marseille, et ont vécu régulièrement dans la région. D'autre part, la variabilité qui a pu être constatée ne saurait empêcher de dégager des tendances nettement affirmées, comme nous allons le voir.

Les problèmes relatifs au conditionnement syntaxique de la liaison et de l'élision ne nous intéressent pas directement ici (concernant ce problème du conditionnement syntaxique, voir Tranel, I987:I71-190). Nous n'examinerons que des exemples où les conditions syntaxiques d'application de ces processus sont réunies. Leur non-application éventuelle est alors due à des causes strictement phonologiques qu'il s'agit de déterminer, et qui nous éclairent sur la nature des principes régissant les changements qui se produisent aux frontières de mot dans la chaîne parlée. En outre, notre étude est limitée aux cas de liaison normalement obligatoires (voir Tranel, I987: I68-I90, et Encrevé, I988:43-50 pour la tripartition liaison catégorique, liaison variable, et liaison interdite).

Après avoir examiné les données, nous passerons en revue certaines propositions censées rendre compte de faits analogues en français standard (voir Fouché, I959, et Léon, I966), et une solution différente sera proposée.

I. PROPRIÉTÉS PHONOLOGIQUES DU FRANÇAIS DE MARSEILLE

Nous n'énumèrerons pas ici la liste des traits caractéristiques de la variété étudiée (pour un enquête détaillée sur les variétés régionales incluant le français du midi, voir Walter, I982). Nous signalerons simplement deux traits, qui ont un rapport étroit avec le problème du sandhi externe: la prononciation du ' $E$ ' (schwa) final des polysyllabes, et la fréquence de la diérèse.

On trouvera ci-dessous quelques informations sur les locuteurs avec qui les conversations ont été les plus fréquentes (tous ont passé l'essentiel de leur existence dans les Bouches-du-Rhône): - M.F.: née à Saint-Chamas en I946. Etudes supérieures. Professeur de lycée.

- R.B.: né à Saint-Maximin en I944. Etudes supérieures. Professeur de collège.

- S.F.: née à Saint-Chamas en I9I4. Etudes secondaires. Sans profession.

- D.F.: née à Saint-Chamas en I943. Etudes supérieures. Professeur de collège.

- V.B.: né à Marseille en I944. Etudes supérieures. Professeur de lycée.

- V.F.: né à Martigues en 1949. Etudes secondaires. Conducteur de cars.

- M. B.: née à Port-de-Bouc en I955. Etudes secondaires. Sans profession.

- R.G.: né à Fos-sur-Mer en 1949. Etudes supérieures. Professeur de collège.
A Marseille et dans sa région, le 'E' final de mot (précédé d'une consonne ou semi-voyelle) est généralement prononcé avant une consonne ou une pause: (I) la petite fille [lapøtitəfijə]

mais il est élidé avant une voyelle:

(2) c'est une grande amie à moi [setynaguãndamiamwa]

On est donc autorisé, en français méridional, à parler d'élision même dans le cas de polysyllabes tels que grande, petite. En revanche, en français non méridional, dans une prononciation non artificielle, le schwa final de polysyllabe est normalement muet, même avant consonne:

(3) la petite fille [laptitfij]

et son apparition est le plus souvent destinée à 'briser' des groupes consonantiques:

(4) (a) un arbre centenaire [ẽnasbьəsãtñ்̃]

(b) un film tchèque [ẽfilmotfદk]

Le deuxième de ces exemples (4b) montre que le schwa qui apparaît dans ces contextes ne correspond pas nécessairement à une réalité graphique (à propos du 'E', voir Tranel, I987:86-107, et surtout, pour le problème du 'E' dans une variété méridionale, Durand, Slater et Wise, I987).

L'autre particularité qui nous intéresse ici est la plus grande tendance à la diérèse, que l'on constate en français de Marseille: le ' $\mathrm{i}$ ' de lion, avion, par exemple, est syllabique, et on prononce [lijãn] [avijõn] (avec un [j] de transition), alors que la prononciation normale en français parisien est [ $[\mathrm{j} \tilde{\mathrm{J}}]$ [avjã] (voir Martinet et Walter, I973:I2I, 53I). La différence s'explique souvent par l'histoire de la langue, et de ce point de vue le français de Marseille est plus conservateur; dans l'étymon latin de lion, par exemple, la source du 'i' est syllabique: leo, leonis, et la séquence VV explique la diérèse actuelle; en revanche, l'étymon de pied (prononcé [pje] même à Marseille) est $p \mathbf{e}, p \mathbf{e}$ dis, avec une voyelle simple qui se diphtonguera plus tard: en général, il y a synérèse en français de Marseille seulement si la source de la séquence semi-voyelle + voyelle est une voyelle simple en latin. Il faut d'ailleurs préciser à cet égard qu'il ne s'agit pas toujours d'une contrainte phonotactique, et que l'on rencontre des oppositions: ${ }^{2}$

$\begin{array}{ll}\text { (5) oui }[\mathrm{wi}] & \text { ouie [ui] } \\ \text { pion [pjõn] } & \text { pillons [pijõn] } \\ \text { pied [pje] } & \text { piller [pije] }\end{array}$

avions [avjõn] avion [avijõn]

(avions $=$ Ière personne du pluriel de avoir)

Malgré l'existence de ces paires minimales, nous verrons plus loin que le nombre de séquences semi-voyelle + voyelle est plus réduit en français de Marseille qu'en français parisien et que les possibilités de combinaison sont plus restreintes dans le midi provençal.

${ }^{2}$ Notons que ouie se prononce [wi] à Paris et avion s'y prononce [avjã] (Martinet et Wáalter 1973: I21, 626). 
2. LES DONNÉES

On trouve deux grands groupes de mots susceptibles d'être impliqués dans les processus de sandhi:

(i) les mots à initiale vocalique;

(ii) les mots à initiale semi-vocalique.

Les termes qui nous intéressent sont surtout ceux qui ne permettent pas à l'élision et à la liaison de s'appliquer: or, les membres de la première classe (à initiale vocalique) qui empêchent l'élision et la liaison sont, entre autres, les mots dits à $\mathrm{H}$ aspiré, depuis longtemps identifiés par les grammaires traditionnelles (Grevisse, I980:73). On sait que dans l'énorme majorité des variétés de français le ' $H$ ' graphique ne correspond à aucun segment phonétique, mais qu'un certain nombre de mots à 'H' graphique initial on comme propriété d'empêcher l'élision et la liaison de s'appliquer, mểme si leur initiale est vocalique d'un point de vue phonétique:

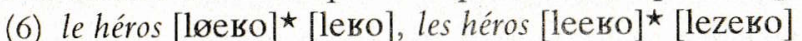

Ces mots sont improprement appelés mots à $\mathrm{H}$ aspiré (concernant le ' $\mathrm{H}$ ', on peut consulter Fouché (I959:25I-265)). La liste ci-dessous comprend des exemples de mots comportant un $\mathrm{H}$ aspiré d'après les règles académiques du 'bon usage', et qui sont apparus dans les conversations:

(7) hache, haie, haine, halte, hameaw, hamster, hanche, hangar, hareng, haricot,

hasard, hausse, haut, hauteur, héros, hêtre, hibou, homard, honte, honteux, housse, hublot, huer, huée, hurler, hutte

Tous ces termes sont aussi traités comme des mots à $\mathrm{H}$ aspiré par les informateurs du dictionnaire de Martinet et Walter (I973:435-458).

On trouvera ci-après en (8) des termes à initiale semi-vocalique dont le comportement en relation avec la liaison et l'élision a également pu être observé. Ils sont classés d'après la nature de la semi-voyelle initiale. Le français (standard ou méridional) comporte trois semi-voyelles (au moins au niveau de la réalisation phonétique): $\left[\begin{array}{lll}j & \text { y } & \mathrm{w}\end{array}\right]$. La semi-voyelle [ 4$]$, qui est une palatale arrondie, est la contrepartie semi-vocalique de [y] (pour les semivoyelles, voir Léon (1966:27-32)).

(8) (a) Mots avec [j] initial:

hiérarchie, hiéroglyphe, hyène

iode, iodé

yacht, yaourt, yeux, yoga, yougoslave, yo-yo

(b) Mots avec [u] initia]

huile, huiler, huissier, huit, huitième, huître

(c) Mots avec [w] initial:

oie, oiseau, oisif, oisiveté

ouate, ouest, oui, onistiti

watt, week-end, western, whisky

Remarque: pour ouest on trouve aussi la variante avec [u] syllabique initial ([uest] au lieu de [west]).
3. ETUDE DES DONNÉES

3. I. Mots à initiale vocalique

\section{I. I. Mots à $H$ aspiré}

Le français de Marseille se démarque de la norme académique, du 'bon usage' (Grevisse, I980), ou encore du français dit standard (Fouché, I959; Léon, I966), de deux façons différentes:

- certains termes que les grammaires traditionnelles classent comme étant à $\mathrm{H}$ aspiré ne se comportent pas comme tels;

- inversement, d'autres termes qui ne sont pas classés dans la liste des mots à $\mathrm{H}$ aspiré bloquent la liaison et l'élision.

Dans la première catégorie, on relève:

(9) haillons, hamster, handicap, handicapé, haricot, hasard, hauteur, héros, Hollande, hurler

Il importe ici d'être nuancé. En effet, d'une part tous les locuteurs ne traitent pas tous les termes de la même façon; d'autre part il faut absolument examiner dans quel(s) contexte(s) on constate une différence entre la norme académique ou le 'bon usage' et le français de Marseille; enfin, ces écarts par rapport à la norme en question sont loin d'être propres au parler étudié ici (le cas typique d'écart répandu sur tout le territoire français étant haricot). Toutefois, tous les termes qui se trouvent en (9) sont des mots à $\mathrm{H}$ aspiré chez Martinet et Walter (I973:435-458). Voici des exemples de séquences qui ont été entendues:

(Iо) tu veux des haricots? [tyvødezariko]

c'est des hamsters [sedezamsteи]

c'est à l'hasard [sealazar]

à quelle hauteur? [akclotœs]

on est allés en Hollande [onetaleanolãndə]

$i(l) s$ hurlent souvent [іzувləsuvãn]

3.I.2. Le problème de l'enchaînement

En ce qui concerne ils hurlent, on peut entendre au moins trois prononciations différentes, aussi bien dans le midi provençal que dans les autres régions:

( I I) ils hurlent: (a) [izysla]

(b) [il?уйә]

(c) [iyslo]

Dans la première variante (I Ia) la liaison ('fautive') s'applique, et la présence $\mathrm{du}[\mathrm{z}]$ de liaison cause généralement la chute du [l] de ils en position préconsonantique (cf. il aime [ilemo], il veut [ivø]):

(I 2) $[$ il увlə $\rightarrow$ ilz yвlə $\rightarrow$ izyslə];

Lorsque la liaison s'applique à cette séquence, il est très rare que le [1] de ils soit maintenu, mais la prononciation [ilzyвlə] est théoriquement possible. 
Dans la deuxième variante ([il’yslə]), qui appartient au registre stylistique le plus élevé, il n'y a pas de liaison, conformément aux règles du 'bon usage', et en outre il n'y a pas non plus d'enchaînement, c'est-à-dire que le [1] final de ils ne subit pas la règle de resyllabification qui, en discours, transfere habituellement la consonne finale fixe d'un mot à l'initiale de la première syllabe du mot suivant, si celui-ci commence par une voyelle:

(I3) il aime $[\$ i 1 \$ \varepsilon \$ m ə \$] \rightarrow[\$ i \$ 1 \varepsilon \$ m ə \$]$

$(\$=$ frontière de syllabe).

Rappelons que l'enchaînement concerne les consonnes finales de mot qui sont toujours prononcées, autrement dit les finales fixes, alors que les consonnes de liaison ne sont pas fixes: elles sont muettes avant consonne ou pause (sur la question de l'enchaînement, voir Encrevé (I988:24 et sequ.)). Dans les cas de non-enchaînement la consonne finale du premier mot de la séquence reste finale de syllabe:

(I4) ils hurlent $[\$ 11 \$$ уь $\$$ lə\$]

et on insère en général une occlusive glottale:

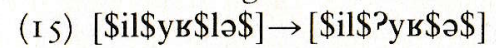

Enfin, dans la troisième variante ([iyrlə]), la chute du [1] de ils est particulièrement intéressante; en effet cette chute se produit normalement en discours spontané, non surveillé, avant une consonne: or le verbe a dans cet exemple une initiale vocalique, d'un point de vue strictement phonétique (on trouvera une explication de ce phénomène en $\$ 5 . \mathrm{I}$ ).

Il est à noter que la prononciation [\$ilys\$la\$], avec enchaînement, ne saurait être interprétée que comme le singulier il hurle, car si un locuteur accepte l'enchaînement dans ce cas, il acceptera a fortiori la liaison au pluriel, et il prononcera [\$izyь\$lə\$]. En revanche, les variantes (I Ib) et (IIC) ci-dessus peuvent être interprétées aussi comme des singuliers (il hurle).

3.1.3. Point de vue fonctionnel ('conspiration')

En fait, l'absence de liaison dans ils hurlent, la chute de [1] (dans la variante [іувlə]), l'absence d'enchaînement et l'insertion de la glottale (dans la variante [il’уувlə]) jouent exactement le même rôle fonctionnel: dans tous les cas on évite la rencontre d'une consonne ([z] de liaison ou [l] final fixe) avec la voyelle initiale ([y]) du deuxième terme de la séquence. En somme, tout 'conspire' à protéger le verbe hurler (sur la notion de 'conspiration' de règles, voir Kisseberth, I970).

Au féminin, le maintien du schwa final du pronom elles joue également le même rôle, et participe à la 'conspiration':

(I6) elles hurlent [عləувlə].

Le locuteur est soucieux de marquer phonétiquement la frontière de mot, quel que soit le moyen utilisé.
3.1.4. H aspiré, accent, et nombre de syllabes

En ce qui concerne l'exemple à quelle hauteur (voir (Io) ci-dessus), prononcé [akelotœь], avec élision du 'E' final de quelle, il est nécessaire de préciser que cela ne signifie pas pour autant que hauteur ne soit pas un mot à $\mathrm{H}$ aspiré en français de Marseille; en effet les mêmes locuteurs prononcent régulièrement la hauteur, les hauteurs [lаotœu], [lеотœь], sans élision ni liaison. Il faut, pour comprendre les faits, comparer à quelle hauteur et quelle honte (voir Dell, I970:86-88; Cornulier, I978; et Tranel, I98 1:307):

(I7) (a) à quelle hauteur [akclotœr]

(b) quelle honte [keləõntə].

Dans le deuxième exemple ( $\mathrm{r} 7 \mathrm{~b})$, le ' $\mathrm{E}$ ' (schwa) final de quelle se maintient toujours: la différence est clairement due (d'autres exemples le prouvent: voir plus loin) à la structure prosodique des mots concernés; hauteur est un dissyllabe dont l'accent lexical, c'est-à-dire l'accent dans la forme de citation du mot, frappe la deuxième syllabe, tandis que honte, bien qu'étant dissyllabique aussi, a l'accent lexical sur la première syllabe (sur l'accent en français, voir Léon, I966:15, et Garde, 1968:94-96). Nous sommes en mesure de postuler le principe suivant:

le schwa final des polysyllabes se maintient plus facilement avant un mot à $\mathrm{H}$ aspiré dont l'accent lexical frappe la première syllabe.

Par conséquent le schwa de quelle se maintient avant honte, mais rarement avant hauteur (les deux termes étant dissyllabiques, le rôle de l'accent lexical à l'initiale ressort clairement). En outre, on observe que le schwa final des dissyllabes se maintient plus facilement (dans les mêmes conditions) que celui des mots de plus de deux syllabes; le schwa final de grande a donc plus de chances de se maintenir qui celui de petite dans des séquences telles que grande harpe [gка̃ndəакрә], petite harpe [рøtitəаврә], [рøtit?аврә]. Dans le second cas, le locuteur marseillais peut préférer insérer une occlusive glottale plutôt que de maintenir le schwa final.

3. I.5. Non-application des règles de sandhi et rôle de l'accent lexical

Le français méridional, tout comme le français parisien (ou les autres variétés non méridionales), traite certains mots, dont l'initiale orthographique n'est pas un ' $H$ ', de la même façon que les mots à $H$ aspiré. Citons notamment:

- les numéraux à initiale vocalique: le un, le onze;

- les sigles: le RPR, le MRG;

- les noms de lettres: la 'a', le 's', le 'l', etc.

On trouve toutefois aussi d'autres exemples, qui different des précédents dans la mesure où ils sont spécifiques des parlers méridionaux:

(I 8) une anse [ynəãsə]

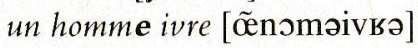




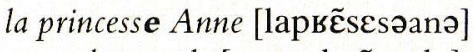

une robe ample [уnәкวbəãmplə]

le noble art [lønobləas]

la ire [Іаівә]

Un facteur prosodique déterminant est ici à l'oeuvre, comme dans les cas qui viennent d'être étudiés (hauteur, honte): la place de l'accent lexical. En effet, on remarque que anse, ivre, Anne, ample, art, et ire sont tous accentués sur la première syllabe dans leur forme de citation; ainsi, à chaque fois, le schwa final (ou le [a] de l'article défini féminin) précède une syllabe qui porte l'accent lexical, ce qui a tendance à faciliter son maintien. La structure prosodique du mot concerné joue donc un rôle crucial; il suffit de comparer: (I9) la princesse Anne [lapке̃sesəanə] (maintien du 'E')

la reine Amélie [lakenameli] (élision)

Le nom Amélie n'est pas accentué sur la première syllabe, et le degré de probabilité de non-application de l'élision diminue.

On pourrait être tenté de penser qu'un autre facteur, la longueur du second mot de la séquence, joue aussi un rôle. En réalité, la place de l'accent lexical et le nombre de syllabes du mot constituent un seul et même facteur, le principe étant le suivant: plus l'accent lexical du deuxième mot de la séquence est proche de la syllabe initiale de ce mot, et moins la liaison et l'élision ont de chances de s'appliquer. Or, l'accent en français frappe la dernière syllabe du mot, sauf si ce mot se termine par un schwa, auquel cas l'accent frappe la pénultième:

(20) 'sport, spor'tif, spor'tive, sportive'ment, etc.

Par conséquent, plus le nombre de syllabes augmente, et plus l'accent lexical s'éloigne de l'initiale. Le nombre de syllabes du second mot de la séquence n'est donc pas en soi un facteur pertinent. En revanche, la longueur du premier mot joue certainement un rôle: plus ce mot est long, et plus l'élision risque de s'appliquer. Ainsi, on pourra constater une variation chez certains

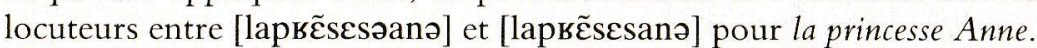

La structure prosodique n'est pas seule en cause, et la fréquence d'un terme est également susceptible de jouer un rôle: moins un terme est fréquent, et moins l'élision et la liaison ont de chances de s'appliquer, toutes choses étant par ailleurs égales; ainsi ire est un mot vieilli et rare, ce qui fait qu'on a deux raisons de le traiter comme un mot à $\mathrm{H}$ aspiré, ces deux raisons étant sa structure prosodique et sa faible fréquence. D'autre part, si la princesse Anne

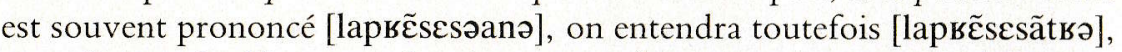
avec élision régulière, pour la princesse entre: ${ }^{3}$ on peut penser que la différence est due au fait que Anne est un nom propre, dont la fréquence est plus faible: Anne fonctionnera par conséquent comme un mot à $\mathrm{H}$ aspiré, contrairement au verbe entrer.

On ne peut que réitérer l'hypothèse émise plus haut $(\$ 3$.I.3): la non-

${ }^{3}$ Je remercie ici Jacques Durand, à qui je dois cette observation. Je suis évidemment seul responsable de l'interprétation des données. application de la liaison et de l'élision constitue un signal démarcatif (négatif), c'est un procédé destiné à 'protéger' le second mot de la séquence, à préserver son autonomie relative dans la chaîne parlée. Le maintien du schwa dans noble art, robe ample, etc. fait de cette voyelle un véritable indice de frontière de mot. Si le français méridional dispose, avec le maintien du schwa final des polysyllabes, d'un procédé démarcatif idéal, les variétés non méridionales, quant à elles, ont recours, moins souvent il est vrai, à un autre procédé démarcatif, qui consiste à insérer une glottale:

(2I) (a) une robe ample [ynəঞəbəãmplə] (variante marseillaise)

(b) une robe ample [упьว วãpl] (variante parisienne)

les deux procédés démarcatifs (maintien du schwa et insertion de la glottale) permettent d'éviter l'enchaînement. La différence entre les deux variétés de français s'explique en grande partie par le fait que le locuteur non méridional prononce [упьэb] avant pause, alors que le locuteur méridional prononce [yпәвəbə] dans le même contexte: on n'est pas autorisé à postuler une règle d'élision de schwa final de polysyllabe en français non méridional, si l'on accepte l'idée que ce schwa final est absent des formes sous-jacentes dans la variété en question.

3.2. Mots à initiale semi-vocalique

3.2. I Séquences attestées à l'initiale

Avant d'examiner ce qui se passe dans les contextes de liaison/élision, il est souhaitable d'inventorier les combinaisons possibles de semivoyelle + voyelle dans le parler de Marseille. Il s'agit de déterminer par quelles voyelles chacune des semi-voyelles $\left[\begin{array}{ll}j & \mathrm{w}\end{array}\right]$ peut être suivie. On trouvera ci-dessous les séquences semi-voyelle + voyelle attestées

(22) avec [j]:

[ji] Yiddish (seul cas à l'initiale)

[ju] yougoslave

[je][je] pied, Hyères

$[\mathrm{j} ø]$ yeux

[jo][jo] iodé, iode

[ja] yoourt

(23) avec $[\mathrm{u}]$ :

[qi] huile

Il est remarquable qu'en français de Marseille, cette combinaison [ui] soit la seule attestée et la seule possible avec la semi-voyelle [4] (écuelle, par exemple, se prononce [ekyclə]; même le mot juin se prononce ordinairement $[3 w \tilde{\varepsilon} \eta]$ plutôt que $[3 \zeta \tilde{\varepsilon} \eta]([u]>[w]))$.

(24) avec [w]:

[wi] whisky, ouistiti

[we][we] fouet, fouette

[wa] oiseau, onate 
Si l'on examine ce qui se passe lorsque les conditions phonologiques d'application des processus d'élision et liaison sont apparemment réunies, on constate que bon nombre de termes bloquent ces processus dans la mesure où on peut encore les considérer comme [+ étranger] (voir $\$ 5.4$ et la note 6 pour le rôle exact de ce trait); voici quelques exemples:

(25) yacht, yaourt, yen, watt, week-end, whisky, etc.

3.2. I. Comportement du yod initial

La non-application de la liaison et de l'élision avant un [j] initial est régulière, chez la grande majorité des locuteurs marseillais:

(26) des hyéroglyphes [dејевоglifə]

des hyènes [dejenə], une hyène [ynəjena]

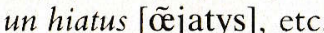

(Il est à noter que hyéroglyphe n'est un mot à $\mathrm{H}$ aspiré pour aucun des informateurs de Martinet et Walter (1973:447), et que hiatus (447) et hyène (456) ont un comportement variable.) Seuls deux mots relativement fréquents font exception (iode et yeux) en acceptant les processus de sandhi:

(27) de l'iode [døljodə]

elle a de beaux yeux [Eladøbozjø]

En ce qui concerne iode, on peut d'ailleurs remarquer que ses dérivés (ioder, iodê) refusent liaison et élision, et se comportent donc régulièrement; quant à yeux, il semble représenter un cas spécial: en effet si on demande aux informateurs quel est le pluriel de oeil, la réponse est plus souvent [zjø] que [jø], la forme [zjø] étant prononcée ainsi même isolément (on note également le dérivé zyeuter!). Il se pourrait donc fort bien que le $[\mathrm{z}]$ fonctionne dans ce cas plutôt comme une consonne initiale fixe que comme une consonne de liaison. Précisions à ce propos que traiter le $[z]$ de yeux comme l'initiale du mot n'entraîne pas que ce $[\mathrm{z}]$ soit un préfixe (la présente hypothèse differe donc de celle de Gougenheim (I938:59-60); en outre, elle ne concerne qu'un seul mot). Si l'analyse est exacte, yeux cesse d'être une exception. On peut énoncer de toutes façons le principe suivant pour le français de Marseille:

La semi-voyelle [j] fonctionne à l'initiale comme les consonnes et non comme les voyelles, pour ce qui est de la liaison et de l'élision.

3.2.3. La combinaison [ui]

Voyons à présent comment se comporte la seule combinaison possible avec la semi-voyelle [u], c'est-à-dire [ui]; les termes suivants acceptent la liaison et l'élision en français standard:

(28) huile, huileux, huiler, huilier, huis, huissier, huitre

D'autres termes refusent ces processus:

(20) huis clos, huit, huitaine, huitième

A Marseille, on observe qui si huile accepte la liaison et l'élision, ses dérivés huileux, huiler la refusent:

(30) tu les huiles ou pas? [tyleuilupa]
Pourtant, huile et ses dérivés n'ont pas de $\mathrm{H}$ aspiré d'après les informateurs de Martinet et Walter (1973: 454). Pour huilier, on constate à Marseille certaines hésitations (la variabilité est inter- et intra-individuelle). La différence de traitement entre huile et ses dérivés (en Provence) ne peut guère s'expliquer que par la faible fréquence de ces derniers (on a vu ci-dessus en $\$ 3.1 .5$ qu'une faible fréquence est un facteur favorisant la 'protection' d'un mot). Si l'on considère uniquement les termes qui sont relativement fréquents, on limite la liste à huile, huissier, huit, huitième, huître; on constate alors que sur ces cinq termes, trois admettent la liaison et l'élision: huile, huissier, huître; les deux autres (huit, huitième) sont des numéraux: or un numéral, même avec une initiale vocalique ou semi-vocalique, refuse la liaison et l'élision (un, une, huit, huitième, onze, onzième bloquent les processus de sandhi: voir Martinet et Walter (I973: 454, 6I 8)). On est donc en droit d'en conclure que [ui] est une séquence phonétique qui, en tant que telle, admet la liaison et l'élision (contrairement à la semi-voyelle [j]).

3.2.4. La semi-voyelle $[\mathrm{w}]$

Il nous reste à examiner la situation des mots avec [w] initial. On trouve les combinaisons suivantes: [wa] [wi] [we]. Nous avons vu plus haut que les mots auxquels on peut attribuer le trait [+ étranger] bloquent la liaison et l'élision:

(31) watt, water-polo, week-end, western, whisky, whist

Il existe cependant deux cas douteux de ce point de vue: oued et ouistiti; oued est un mot arabe, nettement senti comme tel par ses connotations, mais il ne bloque pas les processus de sandhi; ouistiti, dont l'origine est difficile à déterminer avec certitude, bloque ces mêmes processus chez la plupart des informateurs, mais pas tous: pour une minorité, la liaison et l'élision son possibles (variabilité inter-individuelle). Reste le cas des mots non étrangers:

(32) [wa] oie, oiseau (et ses dérivés), oisif, oisiveté, ouailles

(we] ouest

[wi] oui

Le cas de oui est vite réglé; ce terme pose le problème de la liaison et de l'élision le plus souvent en fonction métalinguistique:

(33) Le oui du Président à cette question

Dans ce contexte, la règle générale est la non-application du sandhi. Pour le reste, la règle dominante (mais non générale) pour les mots qui ont le trait [- étranger] est l'application des processus de sandhi externe; en effet ces processus ne sont bloqués que par oisif (mais pas par oisiveté), et seulement chez certains locuteurs. Le cas de ouate est spécial: en français de Marseille, on constate la même variabilité que dans les autres régions, les puristes euxmêmes admettant aussi bien de l'ouate que de la ouate, tout comme le dictionnaire de Martinet et Walter (1973: 625).

Si l'on doit se prononcer en faveur d'une interprétation de ces données, 
on est donc amené à penser que le cas normal, non marqué, lorsque la semi-voyelle initiale est [w], est l'application des processus de sandhi externe.

3.3. Distribution des semi-voyelles en position non initiale de syllabe

3.3.I. Le problème du yod

Nous allons à présent chercher des corrélations éventuelles entre le comportement des séquences semi-voyelle + voyelle à l'initiale du mot en contexte de liaison/élision, et la distribution de ces mêmes séquences en position non initiale de syllabe. Nous savons déjà (voir $\$ 3.2$ plus haut) que les combinaisons semi-voyelle + voyelle sont en nombre plus restreint en français de Marseille qu'en français non méridional.

Ce qui nous intéresse ici, ce sont les combinaisons possibles après des séquences bruyante + liquide (bruyantes=occlusives orales et fricatives; liquides $=[\mathbf{B}]$ et $[1])$; en effet, c'est dans ce contexte qu'interviennent des contraintes d'ordre phonotactique dans toutes les variétés de français. Or, à Marseille, il s'avère que la semi-voyelle [j] n'apparaît jamais après un groupe bruyante + liquide (il se peut que le français de Marseille differe en cela des autre variétés méridionales, mais seul le parler marseillais nous concerne ici). On sait que la diérèse est fréquente en français de Marseille (voir $\$ I$ ); grâce à cela, on peut déterminer que la forme de base du morphème d'imparfait aux Ière et zème personnes du pluriel (nous chantions, vous chantiez) est $/ \mathrm{j} /$ et non /i/: si la forme de base était /i/, elle ne subirait pas de règle de semivocalisation dans un dialecte où la diérèse est particulièrement fréquente; le semi-voyelle [i] qui apparaît à l'imparfait n'est donc pas le produit d'une règle de semi-vocalisation, mais c'est au contraire l'allomorphe de base:

(34) vous aimiez [vuzemje]

vous regardiez [vuвøgaвdje]

Cet allomorphe de base ne peut se maintenir après un groupe bruyante + liquide:

(35) vous prendriez [vиркãndкije].

C'est le processus inverse qui a lieu en fait: l'allomorphe de base / $\mathrm{j} /$ subit une règle le convertissant en /i/ syllabique, et on insère ensuite une semi-voyelle de transition $(\mathrm{j} \rightarrow \mathrm{i} \rightarrow \mathrm{ij})$. Ce qui est pertinent dans le cadre de notre étude est l'impossibilité d'une prononciation * [vирка̃ndкje]. Il s'agit d'une contrainte absolue en français de Marseille; il est bien connu que c'est aussi une contrainte en français standard (voir Léon, 1966:27), mais en réalité on relève en discours rapide, non surveillé, certaines occurrences de séquences bruyante + liquide $+[\mathrm{j}]$, dans la région parisienne notamment (voir Tranel, I987:I2I): cela ne se produit jamais à Marseille.

3.3.2. Le séquence [qi]

Le séquence [ui] est parfaitement prononçable après les groupes de consonnes en question:

\section{(36) truie [tвчі]}

pluie [plui]

Rappelons que cette combinaison [ui] est, en français de Marseille, la seule possible avec la semi-voyelle [4].

3.3.3. La semi-voyelle $[w]$

Enfin, avec la semi-voyelle [w], seules sont attestées après les groupes

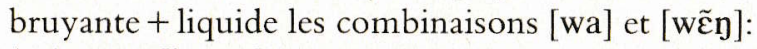

(37) croix [kwwa]

trois [tвwa]

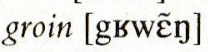

Il faut d'ailleurs noter que la séquence [wa] est en opposition avec la séquence dissyllabique [ua], avec une frontière de morphème avant le - $a$ :

(38) trois [tswa], il trona [iltsua]

loi [lwa], il loua [illua].

En revanche la séquence [4i] n'est jamais en opposition avec [yi], qui est une séquence illicite, même après un groupe bruyante + liquide (on peut entendre cette séquence dans le sigle QI [kyi], quotient intellectuel, mais chaque letter prononcée a alors le statut de mot; la voyelle est donc finale de mot, et la contrainte est inopérante $\left.{ }^{4}\right)$.

Nous devons à présent nous poser le problème des combinaisons [wi], et [we] ou [we]; ces combinaisons ne sont pas attestées après les groupes bruyante + liquide, mais on est en droit de penser qu'il s'agit d'un fait purement accidentel. En effet, aucun des informateurs interrogés n'a éprouve de difficulté à produire des mots forgés tels que ceux qui suivent:

(39) [kвwе], [tвwe], [kвwik], etc.

Si l'on veut bien admettre, pour ce qui est de [w], qu'il y a là un fait de distribution lacunaire plutôt qu'une véritable contrainte phonotactique, on constate alors une corrélation particulièrement intéressante: les combinaisons semi-voyelle + voyelle qui, en tant que telles, ne bloquent pas la liaison et l'élision sont aussi (chez la majorité des informateurs) celles qui sont admises (sinon toujours attestées) après des groupes bruyante + liquide. Il ne peut s'agir d'une coüncidence. En outre ces combinaisons apparaissent également aux frontières de mot, après application des règles d'élision et d'enchaînement:

(40) quatre oiseaux [katbwazo]

quatre huiles [katsuilə]

Si l'enchaînement n'a pratiquement jamais lieu dans des séquences telles que

4 Il est vrai qu'on trouvera la séquence dissyllabique [yi] à l'intérieur du mot, à condition qu'une frontière morphologique précède le $-i$, avec par exemple le suffixe -iste: un partisan de Landru aurait été un landruiste [lãndryista]. On peut postuler que -iste est un suffixe de classe II (voir Siegel, I974; Scalise, I984, et Durand, I990: I70-I73. Dans l'optique de Selkirk (I982b ), on peut dire que -iste est attaché à un mot, et non à une racine: la prononciation du mot 'interne' (Landru) est alors préservée. 
quatre whiskies, c'est bien entendu parce que l'élision n'est pas appliquée, le mot étant [+ étranger]. L'enchaînement n'aura jamais lieu dans le cas d'un [j] initial:

(4I) quatre hiéroglyphes [katвәјевоglifə] (voir $\$ 3.2 .2$ ci-dessus)

puisque le schwa final se maintiendra toujours. On retrouve donc les mêmes latitudes et contraintes combinatoires aux frontières de mot que dans le mot isolé.

\section{EXAMEN DE QUELQUES SOLUTIONS}

\section{I. Analyse de la syllabe en constituants immédiats}

Nous avons essayé de montrer qu'il existe une corrélation entre la distribution des semi-voyelles et les phénomènes de sandhi externe tels que la liaison, l'élision, et l'enchaînement. Cette corrélation est beaucoup plus forte en français de Marseille que dans les variétés non méridionales. On est tenté d'aller plus loin, et d'émettre l'hypothèse que les contraintes distributionnelles et les faits de joncture sont dus à la même cause. Les règles phonotactiques étudiées ci-dessus militent en faveur d'une analyse de la syllabe en deux constituants: l'attaque et la rime (voir par exemple Selkirk, 1982a), la rime comprenant elle-même un noyau obligatoire et une coda facultative. Dans un mot tel que pic, par exemple, le / $/$ / constitue l'attaque, tandis que la rime est constituée du noyau /i/ et de la coda $/ \mathrm{k} /$ :

(42)

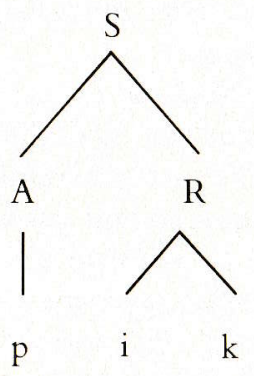

Dans le cadre de cette analyse de la syllabe, la liaison et l'élision ont lieu seulement quand l'attaque est nulle (voir Encrevé, I988, pour les détails). Par conséquent les semi-voyelles bloquant les processus en question sont dans l'attaque, tandis que les semi-voyelles ne les bloquant pas sont dans la rime (le [w] de watt est dans l'attaque, le [w] de oie est dans la rime: voir Tranel, I987:I I 5-I I 8):
$(42)$ watt:

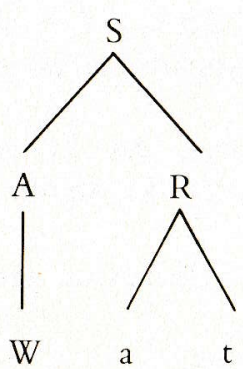

oie:

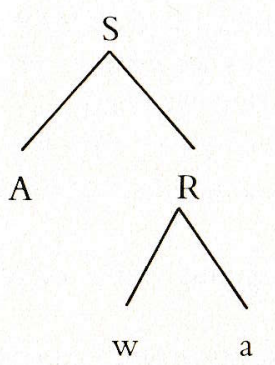

\subsection{L'hypothèse des diphtongues}

Noske (I982:26I-262) adopte une analyse de la syllabe en constituants immédiats. Il note qu'une règle exclut les séquences de trois segments (occlusive + liquide + semi-voyelle) dans l'attaque de la syllabe, mais il

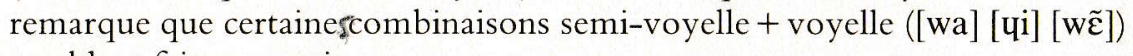
semblent faire exception:

(44) trois, truite, pluie, bruit, groin

L'auteur postule alors que ces combinaisons constituent des diphtongues monophonématiques: toute combinaison semi-voyelle + voyelle susceptible d'apparaître après un groupe bruyante + liquide sera donc automatiquement traitée comme monophonématique, et fera intégralement partie du noyau syllabique (le noyau étant lui-même un constituant de la rime):

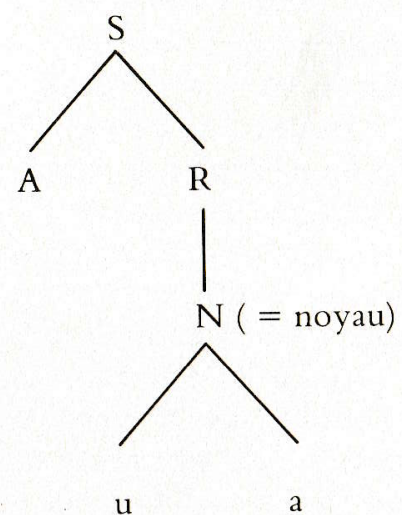

Noske (ibid.) avance toutefois un deuxième argument en faveur de son hypothèse: l'existence d'alternances telles que voir / verra, peux / puisse, tenir / tienne, et il suppose que si une séquence semi-voyelle + voyelle alterne avec une voyelle simple, cette séquence est monophonématique. Plusieurs objections viennent à l'esprit: tout d'abord, ces alternances ne sont guère 
productives, et doivent plutôt être considérées comme des résidus synchroniques de processus historiques; en second lieu, le groupe [je], qui alterne avec une voyelle simple dans tenir / tienne, etc. et qui constituerait donc une diphtongue d'après les critères retenus, n'apparaît précisément jamais après un groupe bruyante + liquide.

Une hypothèse assez proche est avancée par Tranel (I987:IIS-II8, I73-I74), qui postule aussi que l'on a affaire à des diphtongues dans les cas suivants:

- quand la séquence semi-voyelle + voyelle suit un groupe occlusive + liquide;

- quand une semi-voyelle permet la liaison.

Ainsi oiseau, qui accepte la liaison/élision, aurait une diphtongue initiale, mais whisky non, dans le mesure où il refuse les mêmes processus. Lorsque la semi-voyelle est un élément de diphtongue, elle fait partie du noyau, tandis que dans les autres cas elle fait partie de l'attaque de la syllabe.

\subsection{Critique de l'hypothèse}

La vision des choses présentée ci-dessus pose moins de problème en français méridional qu'en français standard, dans la mesure où dans la variété régionale qui nous intéresse on trouve davantage de corrélations entre les deux ordres de fait, à savoir les phénomènes de sandhi et la distribution des semi-voyelles dans la syllabe; en revanche en français standard, les choses sont moins nettes: hiéroglyphe, par exemple, absent de la liste des mots à $\mathrm{H}$ aspiré chez Grevisse (1980:73-74) aussi bien que chez Martinet et Walter (1973:447), ne bloque pas la liaison (au moins pour certains locuteurs non méridionaux) alors que le groupe [je] n'est pas possible après un groupe bruyante + liquide. De plus, pour une séquence donnée, on ne peut prédire de façon certaine si la semivoyelle fera partie de l'attaque ou de la rime: selon cette hypothèse, le $[\mathrm{w}]$ de oiseau, qui accepte la liaison et l'élision, fait partie de la rime, mais celui de $w a t$ fait partie de l'attaque (puisque watt bloque les mêmes processus); le [w] de onate fera partie de l'une ou l'autre selon que le locuteur prononce l'ouate ou la ouate. On note en outre que la différence de structure qui est postulée n'entraîne absolument aucune différence dans la réalisation phonétique: seul l'entourage du mot est affecté, et la différence structurelle joue donc un rôle purement diacritique. Que dire aussi de termes tels que poids [pwa]? Cette fois la séquence [wa] n'est pas initiale, et nous ne disposons d'aucun indice sûr nous permettant de décider si [w] est dans l'attaque ou dans la rime.

\subsection{Le problème de huit}

On peut énumérer d'autres inconvénients de cette approche: tout d'abord, l'une des motivations de l'analyse de la syllabe en deux constituants immédiats (attaque + rime) est l'absence de contraintes distributionnelles liant les segments de l'attaque à ceux de la rime; c'est l'argument invoqué par Halle et Vergnaud (I980:93): 'Our studies have uncovered many phonological processes where the constituents of the syllable - in particular, the onset and rimes - function independently of each other'. Or, certains partisans de ce type d'analyse postulent pour $\mathrm{H}$ aspiré une 'attaque vide non nulle' (Encrevé, I988:1987; cf. aussi Clements et Keyser, I983:I08, pour une approche analogue dans un cadre théorique différent); en d'autres termes on postule une position vide correspondant à ce qu'on appelait autrefois un phonème latent (voir par exemple Malmberg, I972:140). On se retrouve alors confronté à un problème d'indétermination dans le cas d'un terme tel que huit [yit], qui refuse la liaison et l'élision; on a en effet le choix entre deux solutions: (i) la semi-voyelle fait partie de la rime (= [-uit]), et elle est précédée d'une attaque vide non nulle (comme dans le traitement des mots à $H$ aspiré dont l'initiale est vocalique); (ii) la semi-voyelle fait partie de l'attaque (elle n'appartient donc pas au même constituant que la voyelle: [4-] est l'attaque, [-it] est la rime). Encrevé (1988:200) opte pour la deuxième solution (son exemple étant huis clos). Le problème est que la semi-voyelle [u] ne peut être suivie que de la voyelle [i] en français de Marseille: dans l'analyse de la syllabe défendue par Encrevé, cette contrainte n'est plus exprimable pour des mots tels que huit, huis clos, si on postule que la semi-voyelle est dans l'attaque et la voyelle dans la rime, puisque les contraintes phonotactiques sont pour l'essentiel internes à chaque constituant de la syllabe; il est clair que les affinités qui lient la semi-voyelle [u] à la voyelle [i] nous empêchent d'imaginer qu'elles ne se trouvent pas dans le même constituant, même si la théorie autorise par ailleurs des contraintes inter-constituants (rappelons que, dans cette analyse de la syllabe française, la semi-voyelle $[u]$ est possible dans pluie après un groupe bruyante + liquide précisement parce que, ne faisant pas partie de l'attaque, elle n'est pas soumise à la contrainte barrant une semi-voyelle après le groupe en question). En outre huit bloque la liaison (y compris dans cent huit), mais pas dans des composés comme dix-huit [dizuit]: postulera-t-on une structure syllabique différente pour le même mot quand il entre dans la formation d'un composé? (Le même problème est posé par huis, qui accepte l'élision, et huis clos qui la refuse.)

\section{UNE SOLUTION DIFFÉRENTE}

\section{I. Positions phonémiques et positions démarcatives}

Les critiques que nous venons de formuler à l'encontre de l'analyse de la syllabe en deux constituants (voir ci-dessus) nous amènent à proposer certaines révisions.

Supposons, dans le cadre d'une théorie volumétrique (telle que celle 
d'Encrevé, I988), que le squelette soit constitué d'une série de positions, et symbolisons chaque position par un ' $\mathrm{x}$ ':
(46) pic:

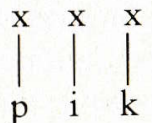
$\mathrm{p}$ i $\mathrm{k}$

Le phénomène de $\mathrm{H}$ aspiré peut s'expliquer par la présence d'une position initiale démarcative, symbolisée par \#:
(47) hasard:

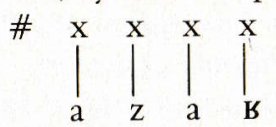
a $\left.\left.\right|_{\mathrm{Z}}\right|_{\mathrm{a}} \mathrm{B}$

Cette position initiale \# a pour effet de bloquer les processus de liaison, d'élision, et, dans la plupart des cas, l'enchaînement ${ }^{5}$. L'avantage d'une telle conception est qu'elle distingue les positions phonémiques (' $x$ '), occupées par des segments à fonction distinctive, et les positions démarcatives ('\#'), ces dernières n'étant permises qu'à l'initiale du mot. Entre consonne et voyelle, la position \# peut être remplie par une occlusive glottale:

(48) sept héros [set?ево]

On a ainsi une explication des phénomènes observées en $\$ 3.1 .2$ : si la structure sous-jacente du verbe hurler est /\#увl+e/, la liaison et l'enchaînement ne s'appliquent pas; on remarque aussi que la position \# peut avoir pour effet de causer la chute du [1] de il(s) dans il(s) hurlent (voir $\$ 3.1 .2$ ), tout comme une consonne initiale: la chute du [l] est en fait provoquée par une attaque non nulle. Quant aux locuteurs qui font la liaison dans ils hurlent, ils ont comme forme sous-jacente $/ \mathrm{ybl}+\mathrm{e} /$, sans position \# à l'initiale.

Dans l'interprétation qui est proposée ici, la glottale n'est donc pas considérée comme la réalisation d'un phonème consonantique initial latent; d'ailleurs, cette occlusive glottale se retrouve dans de nombreux cas de non-enchaînement, que l'on ait affaire à un mot à $\mathrm{H}$ aspiré ou non (voir Tranel, I98I: 3IO-3II).

La position \# précèdera aussi, en français de Marseille, des mots tels que ire, anse, etc. On la trouvera également avant les noms de lettres, et avant les sigles, entre autres (voir \$3.I.5). Cette position peut être sous-jacente, c'est-à-dire faire partie de la représentation lexicale du mot, comme dans tous les cas précités, mais elle pourra aussi être insérée en discours, dans les cas de non-enchaînement pour des raisons rhétoriques (voir Lucci, 1983):

(49) cet événement [sct?evenəmãn]

Dans cet exemple, l'occlusive glottale est la réalisation d'une frontière \# insérée, et non sous-jacente.

5 La solution préconisée ici est proche de celle de Cornulier (1978). Le cadre théorique est toutefois différent. Notons aussi que le symbole \# est ici un signe démarcatif, et ne doit pas être confondu avec les frontières de mot des théories linéaires.
5.2 Contrainte sur l'analyse de la syllabe

Revenons à présent au problème de l'analyse de la syllabe. Afin d'éliminer les inconvénients énumérés ci-dessus, on peut proposer la contrainte suivante:

pour une même séquence segmentale, on ne peut postuler deux structures syllabique différentes si à la différence structurelle ne correspond aucune différence de prononciation de la séquence en question.

En vertu de cette contrainte, un mot tel que onate aura toujours la même structure syllabique, qu'il accepte ou non l'élision (l'ouate, la ouate), puisque sa prononciation est invariante. Une autre conséquence est que le [w] initial de oiseau, qui accepte la liaison et l'élision, et celui de watt, qui les bloque, se trouveront tous deux dans la rime, étant donné que les prononciations des séquences [wa] sont identiques.

\subsection{Le statut du yod}

Les régularités distributionnelles que nous avons examinées révèlent que deux semi-voyelles en position pré-vocalique font partie de la rime: [w] et [u], tandis que la semi-voyelle [j], dans le même contexte, fait partie de l'attaque.

Un autre argument, d'ordre distributionnel également, peut être invoqué en faveur de cette interprétation des données qui accorde à [i] un statut différent de celui des deux autres semi-voyelles: seul [i] est possible après une voyelle tautosyllabique; on trouve en effet des mots avec [j] final: (5o) soleil [solej], ail [aj]

On est en droit de penser que [j], dans ces exemples, joue à nouveau le même rôle qu'une consonne du point de vue distributionnel car, dans ce contexte, il ne commute qu'avec des consonnes:

(5I) ail [aj], art [ав], as [as]

\subsection{Contexte des processus de sandhi}

Le contexte des règles de liaison est évident: ces processus s'appliquent quand l'attaque de la syllabe suivante est nulle; si l'attaque (non nulle) est occupée par \#, les processus sont bloqués; dans le cas d'une initiale semi-vocalique, seul [j] empêchera la liaison et l'élision de s'appliquer, puisque ce segment est la seule semi-voyelle faisant partie de l'attaque. Il nous reste à expliquer pourquoi les processus de sandhi externe ne se produisent pas avant whisky, watt, huit etc. Nous disposons pour cette série de termes d'une possibilité qui est déjà utilisée pour les mots à aspiré: la frontière démarcative (\#). Il est à noter que certaines généralisations sont possibles: les mots [+ étranger] ont automatiquement une frontière \# initiale, ${ }^{6}$ ainsi que les numéraux tels que 6 Durand (I990: 240-24I, n.5) critique de façon convaincante l'usage du trait [+étranger]. Toutefois, dans l'analyse que je défends, ce trait n'est pas directement responsable de non-application des règles de sandhi. On se contente de constater la régularité suivante chez les 
un, huit, onze. A vrai dire, très peu de mots à initiale $[\mathrm{w}]$ ou $[\mathrm{u}]$, et dont la fréquence ne soit pas négligeable, échappent à ces généralisations (même ouate, dont le comportement est variable, pourrait être traité en fait soit comme [+étranger], soit comme [-étranger], selon les locuteurs; son étymologie est d'ailleurs fort douteuse).

\section{CONCLUSION}

L'examen des données montre qu'il est possible de déterminer quels facteurs favorisent la non-application de la liaison et de l'élision avant un mot à initiale vocalique; cet examen révèle aussi clairement qu'il existe en français de Marseille des corrélations entre les processus de sandhi externe (liaison, élision, notamment) et la distribution des semi-voyelles dans la syllabe. Ces corrélations sont plus fortes en français de Marseille qu'en français standard. L'analyse de la syllabe en deux domaines (attaque + rime) permet de rendre compte des faits de façon adéquate, mais il est nécessaire de lui apporter certaines modifications et de limiter le nombre de solutions possibles à l'aide d'une contrainte appropriée. Les trois semi-voyelles ne se comportent pas de la même façon: $[\mathrm{w}]$ et $[\mathbf{u}]$ ont davantage d'affinités avec les voyelles, tandis que [j] se comporte en fait comme une consonne à plusieurs égards. Nous pouvons en conclure que des trois semi-voyelles, seul le yod ([i]) est permis dans l'attaque de la syllabe (et dans la coda). Nous proposons également de considérer que le $\mathrm{H}$ aspiré, et, plus généralement, l'initiale des mots qui bloquent de façon exceptionnelle les processus de liaison et d'élision, correspondent à une position démarcative (\#) dans les formes sous-jacentes. Cette position peut être suivie d'une voyelle, de [w], ou de [u], autrement dit de tout segment susceptible de se trouver à l'initiale de la rime.

Author's address: Université de Provence,

Centre des Lettres et Science Humaines,

29 Avenue Robert Schuman 13621,

Aix-en-Provence,

CEDEX 1 ,

France.

REFERENCES

Clements, G. N. et Keyser, S. J. (1983). CV Phonology. A Generative Theory of the Syllable (Linquistic Inquiry Monograph 9). Cambridge, Mass.: MIT Press. Cornulier, B. de (I978). H aspiré et la syllabation: expressions disjonctives. In: D. Goywaerts (ed.), Phonology in the 1970's. Gand: Story-Scientia.

mots à initiale semi-vocalique: [+étranger] $\rightarrow \#$; cette régularité semble bien affecter les emprunts relativement récents: un one-man show, etc. et les noms propres étrangers: la ville de Onagadougou, etc.
Dell, F. (1970). Les Règles phonologiques tardives et la morphologie dérivationnelle du francais. $\mathrm{PhD}$ thesis, MIT.

Durand, J. (1986). French liaison, floating segments and other matters in a dependency framework. In: J. Durand (ed.), Dependency and Non-Linear Phonology. London: Croom Helm, pp. I6I-20I.

Durand, J. (1990). Generative and Non-linear Phonology. London and New York: Longman.

Durand, J., Slater, C. et Wise, H. (1987). Observations on schwa in Southern French. In: C. Slater, J. Durand et M. Bate (eds.), French Sound Patterns:

Changing Perspectives (Occasional Papers of the University of Essex, Association for French Language Studies, No. 32), pp. 7I-103.

Encrevé, P. (1988). La Liaison avec et sans enchaînement, phonologie tridimensionnelle et usages $d u$ français. Paris: Seuil.

Fouché, P. (1959). Traité de prononciation française. Paris: Klincksieck.

Garde, P. (I968). L'accent. Paris: PUF.

Gougenheim, G. (1938). Le Système grammatical du français moderne. Paris:

Bibliothèque du français moderne.

Grevisse, M. (1980). Le Bon Usage. Paris: Duclos

Halle, M. et Vergnaud, J. R. (I980). Three dimensional phonology. Journal of

Linguistic Research, I: 83-105.

Kisseberth, C. W. (1970). On the functional unity of phonological rules. Linguistic Inquiry, I: 291-306.

Léon, P. (I966). Prononciation du français standard. Paris: Didier.

Lucci, V. (I983). Prosodie, phonologie et variation en français contemporain.

Langue française, 60: 73-84.

Love, N. (1981). Generative Phonology. A Case-Study from French. Amsterdam: John Benjamins B.V

Malmberg, B. (1972). Phonétique française. Malmö: Hermods.

Martinet, A. et Walter, H. (I973). Dictionnaire de la prononciation française dans son usage réel. Paris: France-Expansion.

Noske, R. (I982). Syllabification and syllable changing rules in French. In: H. van der Hulst et N. Smith (eds.), The Structure of Phonological Representations. Dordrecht: Foris, part II, pp. 257-3 IO.

Scalise, S. (1984). Generative Morphology. Dordrecht: Foris.

Schane, S. A. (1968). French Phonology and Morphology. Cambridge, Mass.: MIT Press.

Selkirk, E. O. (1982a). The syllable. In: H. van der Hulst et N. Smith (eds.), The Structure of Phonological Representations. Dordrecht: Foris, part II, pp. 337-383.

Selkirk, E. O. (1982b). The Syntax of Words. (Linguistic Inquiry Monograph 7). Cambridge, Mass.: MIT Press

Siegel, D. (I974). Topics in English Morphology. PhD thesis, MIT.

Tranel, B. (198I). Concreteness in Generative Phonology. Berkeley and Los Angeles: University of California Press.

Tranel, B. (1987). The Sounds of French. An Introduction. Cambridge: Cambridge University Press.

Walter, H. (1982). Enquête phonologique et variétés régionales du français. Paris: PUF. 